\title{
Digital Mont'e Prama: 3D cultural heritage presentations in museums and anywhere
}

\author{
Marcos Balsa Rodríguez, Marco Agus, Fabio Bettio, Fabio Marton, Enrico Gobbetti \\ CRS4 Visual Computing, Pula, Italy - http://www.crs4.it/vic/
}

\begin{abstract}
We present an interactive visualization system developed for the valorization of an extraordinary collection of protostoric Mediterranean sculptures, which depict models of buildings (cone-shaped stone towers), as well as larger-than-life human figures. The architecture is based on scalable components for efficient distribution and adaptive rendering of extremely detailed surface meshes, as well as a simple and effective interactive camera controller tailored for touch interaction. The user interface has been designed for targeting both small screens and large display systems, and in a way that casual users can easily and naturally explore the models with fast learning curves. Furthermore, a thumbnail-based point-of-interest selector enable users to explore 3D views with information presented as 2D overlays decorating the 3D scene. The system components have been integrated in different interactive applications, ranging from large-screen museal setups and low end mobile devices both with very high visual quality. The capabilities of the museal systems have been demonstrated in a variety of temporal and permanent exhibitions, where they have been extensively used by tens of thousands of visitors.
\end{abstract}

\section{INTRODUCTION}

The continuous improvement and proliferation of interactive digital media is reshaping our education and information landscape. Museums are evolving into one of the principal components of our leisure and education industry and, in doing so, are faced with many challenges arising from the competing needs of attracting large masses of visitors while preserving their specificity with an appropriate balance between leisure and learning. At the same time, people is continuously connected to a vast information landscape through mobile devices and pervasive high-speed Internet. In the context of Cultural Heritage, the use of highly detailed 3D models is often necessary to be able to fully represent and present the peculiarities of artifacts. In this sense, the idea of e-tangible collection is growing in the cultural heritage community. In order to fully cover the pre-visit (documentation), visit (immersion) and post-visit (emotional possession) phases [1] of cultural tourism, it is important to create integrated distribution and presentation ecosystems, capable to let users inspect cultural artifacts in both highly spectacular museum settings (e.g., large projection systems), as well as "on the move", using the capabilities of nowadays mobile terminals. Thus, the challenges for digital technologies and technology design are to provide tools that will play a leading role in key issues such as providing access, increasing interaction, sharing knowledge and increasing the commercial viability of heritage institutions [2]. To this end, adaptive systems for easily and naturally exploring and discovering 3D high resolution Cultural Heritage scenes and models are strongly solicited. In this paper, we present the framework developed for the Digital Mont'e Prama project, which aims to present to the public a large unique collection of pre-historic statues of the Mont'e Prama complex, including larger-thanlife human figures and small models of prehistoric buildings (cone-shaped stone towers). The overall complex is composed of 38 reassembled statues, and currently subdivided in two groups, available to visitors in the National Archeological Museums of Cagliari and Cabras. The project covered all 3D digital aspects ranging from acquisition and processing [3], [4] to exploration and rendering [5]. Here we describe our scalable exploration software architecture developed for the project which supports, in an integrated manner, distribution and rendering of massive annotated detailed models with high visual quality. The system enables ubiquitous distribution and visualization on mobile devices, as well as large scale visualization in museum setups. The main components of the system are:

- a framework for interactive exploration of massive highly detailed 3D models of sculptures, adapted for rendering on various scale display, ranging from mobile systems to large projection setups;

- a natural 3D control interface decorated with thumbnail-based point-of-interest selector, adapted to work with various scale displays;

- an infrastructure for authoring and presenting 2D annotation overlays over 3D models and scenes.

Thanks to our framework, object-aware user-interface components are capable to support models composed of various billions of primitives and large numbers of points of interest on either large scale projector systems and mobile devices, and have been used by tens of thousands of inexperienced users in real exhibitions showing the collection of Mont'e Prama sculptures.

\section{RELATED WORK}

Creating interactive systems for the visualization of annotated massive 3D models, in particular for cultural heritage, requires handling large amounts of geometry while providing the user with a user interface that enables both global and indetail inspection, and providing some additional information in order to better comprehend the artifact. Here, we discuss the works which most closely relate to our method.

Massive model rendering. There is a vast literature covering massive 3D model visualization [6], [7], but only recently realtime performance has been demonstrated on mobile devices for very large surface models [8]-[14]. We have adopted the solution proposed by Balsa et al. [11] which adapts the wellknown Adaptive TetraPuzzles [15] to be used in networked environments using mobile devices. 

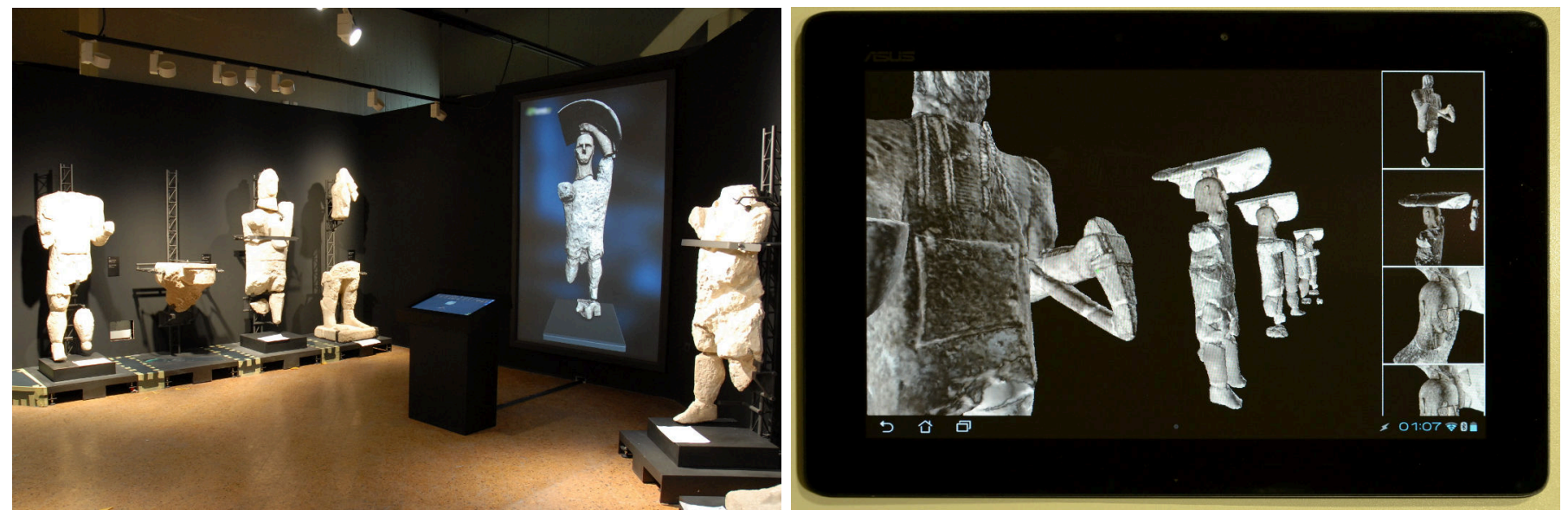

Fig. 1. Digital Mont'e Prama project. Left: permanent museal exhibition setup with a large projector display, together with a small group of real statues; Right: a collection of statues visualized on an ASUS Nexus 7 tablet.

Natural 3D interaction. In terms of complex scene exploration, most of the work in the area is connected to camera/object motion control [16]. Constrained viewpoint navigation has been proposed as an option to reduce the degrees of freedom in order to simplify the access to the user interface for novice users [5], [17]. An automatic pivoting method has been presented [18], but the method requires access to the depth buffer, which is not always available on mobile platforms, and suffers from discontinuities when considering models with sharp features. Our work extends to both co-located and not colocated interaction the approach recently presented by Balsa et al. [19] on auto-centering virtual trackballs for small screens.

Image-assisted navigation.. Image-assisted navigation has been implemented in a variety of ways in the literature. Images can be linked to viewpoints when navigating in a 3D scene, or can also be linked to additional information by using images as hot-spots [20]. Some authors have proposed to hierarchically cluster images according to some image semantic, like combining time and space [21], spatial image-distances [22], or a mixture of them [23]. Our approach is navigation-oriented, presenting the user with a selection of the best candidate views from the current view position. Thus, the user can at any time use the image-based navigation to travel to another point of view.

Information visualization. Using linked multimedia information to enhance the presentation of complex data has been long studied, mostly focusing on guided tours [24], text disposition and readability [25], [26], usability of interaction paradigms [27], and the integration of interconnected text and 3D model information with bidirectional navigation [28]-[30]. Most methods require precise picking to navigate through the information, thus presenting problems when targeting non co-located interaction setups (e.g., large projection displays), and often introduce clutter in the 3D view to display the pickable regions. The solution presented here is based on a method which presents contextual information associated to regions of interest in selected viewpoints, reachable with a thumbnail-based point-of-interest interface without requiring precise picking. A similar approach has been presented by Marton et al. [5] for large-scale setups, and by Balsa et al. [19] for mobile systems. A follow-up work, not described in this paper, concerns techniques useful to provide more structure (e.g., temporal ordering) among annotations, as well as implicit presentation methods based on recommendation systems [31].

\section{CUltural HeRitAgE APPLICATION DOMAIN}

The main motivation of this work was the valorization of the Mont'e Prama complex, which is a large set of extraordinary sandstone sculptures created by the Nuragic civilization in Western Sardinia [32]. The complex was casually discovered during plowing in 1974, and it appeared soon evident that these were truly unique sculptures, displaying features related to both the indigenous Sardinia Nuragic civilization as well as the wider world of the Phoenician colonizers. Four archaeological excavation campaigns were carried out between 1975 and 1979, and revealed five thousands of sculpture fragments which, according to the most recent estimates, comes from a total of life-size 44 statues depicting archers, boxers, warriors and models of prehistoric buildings. With respect to dating, they can be traced to an as-yet undetermined period, which goes from the tenth to the seventh century BC. Restoration was a complicated and time-consuming process: it was carried out at the Centro di Restauro e Conservazione dei Beni Culturali $(C R C B C)$ of Li Punti (Sassari, Italy) and resulted in the partial reassembly of 25 human figures with height varying between 2 and 2.5 meters, and 13 approximately one-metersized building models. Modern non-invasive restoration criteria were considered for the reassembly. No drilling or insertions were performed into the sculptures, fragments were glued together using a water-soluble epoxy resin, and all the gaps on the resin-filled surface were covered with lime-mortar stucco. Furthermore, custom external supports were designed to ensure stability to all the components of the statue, reduce contacts and maximize visibility. The digital valorization project consisted of a long term scientific and technological research plan on the 3D digitization of the complex, aimed at covering all the phases, from the acquisition and processing to the application of innovative methodologies for multiresolution storage, distribution and display. The acquisition process resulted in 37 quarter-millimeter resolution colored surface models [3], [4]. The rest of the paper describes the architecture developed for the distribution and real time interactive exploration of the models on commodity platforms, and for museal exhibitions. 


\section{SYSTEM REQUIREMENTS}

The design of our system has been guided by the requirements collected from domain experts, as well as our analysis of the related work (see Sec. II). Additional requirements were added from our past experience developing interactive systems for cultural heritage [11], [33]. Below we summarize the main requirements that were derived from our analysis for the design of the different system components. See also our previous work for an account of requirements gathered in the Digital Mont'e Prama Project concerning user interfaces [5], [19] and information presentation [31].

\section{A. Distribution and display}

[R.DD.1] High-resolution geometry.. Cultural heritage artifacts generally present information at multiple scales (i.e. global shape and carvings). In particular, the micro-structure typically carries valuable information on the carving process. The Mont'e Prama statues, in fact, present millimeter-sized carvings, thus requiring sub-millimetric model precision.

[R.DD.2] Large-scale visualization.. The imposing size of many artifacts (i.e. statues) has to be transmitted to the visitor for him to have a correct comprehension of the original work. In order to better represent the aura of the statue, in museal exhibitions large displays which reflect the size of the represented object are needed.

[R.DD.3] Display flexibility.. In order to cover the whole pre-visit, visit, and post-visit phases, one should support a wide range of setups, including museum setups, as well as smartphone and tablet applications. The specific application case for our designs is the presentation of larger-than-life human statues, reaching over $2.5 \mathrm{~m}$ of height. The statues were constructed at this imposing scale on purpose, and this macrostructure information should be immediately conveyed to the visitor through a real-scale (or larger-than-real) presentation. This means, in particular, that in museum setups we need to support large (wall-sized) displays.

[R.DD.4] Public accessibility (ubiquity).. Nowadays, smartphones and tablets have become affordable enough as to be present everywhere. Furthermore, the connection bandwidth and performance of those devices are continuously increasing at fast paces, thus providing a great platform for media distribution. So, not only to empower the public access to the information, but also to improve the visit experience (previsit and post-visit phases), the pervasiveness of mobile devices should to be exploited.

[R.DD.5] Low bandwidth usage.. The interactive exploration of those huge models produces a substantial amount of data traffic. In order to provide an interactive and pleasing exploration experience to the user the size of the transmitted information has to be aware of the bandwidth capabilities of the targeted systems (i.e. WIFI and 3G/4G connections).

\section{B. User interface}

[R.UI.1] Single user control, multi-user fruition.. Museums have large amounts of visitors willing to inspect the various collections exhibited. Therefore, enabling multiple visitors to observe the display while the user in control of the interface inspects the object, facilitates the flow of visitors to access visual information faster. In such a way, it will be improved both the visitor experience, since wait time is much less, and the throughput of visitors, which permits more visitors to access the museum installations.

[R.UI.2] Seamless interactive exploration and zooming.. The high resolution geometry present in the 3D representations requires the user to be able to reach viewpoints all around the model, both from far and from very close distances. The exploration, therefore, has to permit a seamless transition from global shape visualization to close detail inspection (i.e. carvings).

[R.UI.3] Fast learning curve and assisted navigation.. Museums receive large amounts of visitors, so any kind of training taking long time is not practical. Hence, the user interface is required to be immediately accessible, allowing the user to inspect the artifact both by directly trying and understanding the user interface, or by using some assisted navigation that gives some hints and/or shortcuts to explore the object.

\section{Information presentation}

[R.IP.1] Focus on work of art.. The visitor intention is on improving his knowledge on the artifact being inspected. So, the user interface needs to be unobtrusive and avoid any distracting widgets, or occlusion from other visitors. At the same time, it should provide the user with an easy and direct way to get to the information without requiring complicated interactions.

[R.IP.2] Information authoring.. Textual and visual information (drawings, images) should be supported. Editing should be made possible for museum curators and archaeologists without particular training. Adding annotations and linking them should not require intervention of specialized personnel.

[R.IP.3] Information spatially connected with 3D models.. Most of the information, textual and visual, is spatially connected to a region of a 3D model. This implies that descriptive information should be associated to parts of the cultural objects, typically seen from a canonical point of view, or at least close to it. Examples are descriptions of carvings and decorations, reconstruction hypotheses, comparisons with other objects. Different macro-structural and micro-structural views should be associated with different kinds of information.

[R.IP.4] Engaging experience.. In general, visitors do not want to be overloaded with instructional material, but to receive the relevant information, learn, and have an overall interesting experience, which should be be personal, selfpaced, and exploratory. The user interface should provide guidance, while not being perceived as overly obtrusive.

\section{SYSTEM COMPONENTS}

We present in this section our proposal to cope with the requirements derived from our analysis of the domain. The nowadays pervasive use of touch devices, such as smartphones and tablets, has made people used to touch interfaces based on direct manipulation of on-screen objects (e.g. 2D maps or 3D games on smartphones). Furthermore, large touch-based displays, such as touch-enabled monitors or wall displays, are increasingly being used to enable users to explore $3 \mathrm{D}$ 


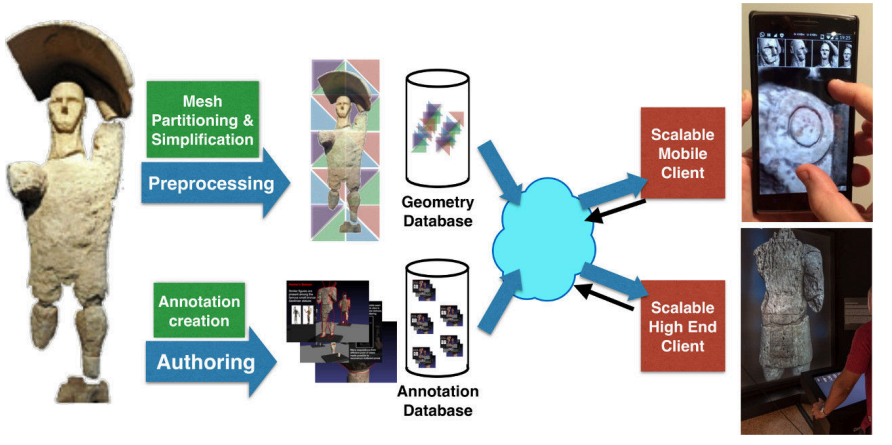

Fig. 2. Architecture components. Pre-processing and server components are shared between the mobile and high-end clients. User interface is implemented on touch devices.

models [34], also in museum installations [5]. Our system is based on a touch interface for the exploration of highly detailed 3D objects using a multiresolution framework for interactive rendering and networked components for streaming the required data. The main components of the framework are represented in Fig. 2.

\section{A. Scalable rendering and distribution}

Real-time rendering of large datasets at high resolution, that is billions of triangles and hundreds of points of interest per model, requires dealing with two main problems: the complexity of the model, and the amount of data to be transmitted and loaded into core memory. Dealing with such complex models requires specialized multiresolution structures and adaptive algorithms. Our rendering framework, and also the user-interface subsystem, exploit a multiresolution representation of the $3 \mathrm{D}$ models. The Adaptive TetraPuzzles (ATP) approach has been extended with all the geometric queries required to compute the automatic pivot, and kd-trees to organize the points of interest. Thanks to the adaptive rendering techniques only a small subset of the dataset is required to provide a high quality representation of the object, so real-time performance is achieved for all scale views (i.e. global shape or close detail) in the various systems presented. In order to cope with the low resource availability on mobile devices, we have used a compact representation that encodes position, normal and color into 64 bytes, thus better scaling on devices with low memory available, while still providing high visual quality. In addition, this compact representation is further compressed for data streaming, to reduce bandwidth requirements and so improve the refinement speed, and the number of triangles per node has been increased from $8 \mathrm{k}$ triangles to $16 \mathrm{k}$ triangles in order to improve streaming performance by reducing the number of packets to transmit.

\section{B. User interface}

According to requirements described in section IV, we designed our user interface components in a way that they could be easily adaptable to various configurations, and easily usable with fast learning times by casual users. Furthermore, we wanted to keep the interface as less distracting as possible, in order to keep the main focus of users to the details of sculptures and the information associated. The main components of our user interface are a selection widget for easily navigating through the entire sculpture collection, an objectbased assisted system for camera control tailored for both orbiting and proximal object inspection, and an image-based navigation component for navigating amongst a series of predefined viewpoints and displaying associated information in overlay.
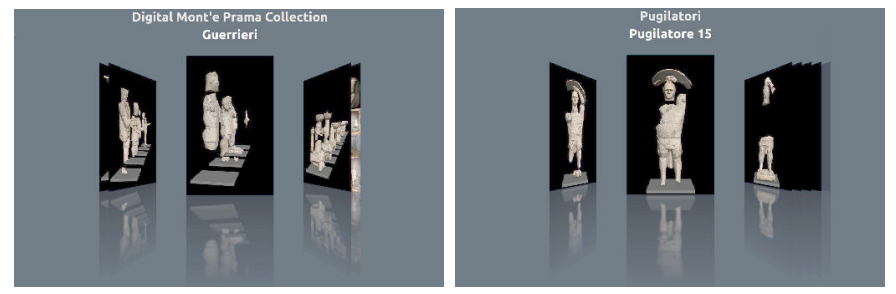

Fig. 3. Sculpture selection widget. The main interface is organized as two level scroll interface hierarchies. The Mont'e Prama sculpture collection is subdivided in 4 categories: boxers, archers, warriors and building models(see left). Selecting one of the group, the sculptures of given category are presented (see right), and can be chosen.

Sculpture selection widget. In order to enable casual users to easily navigate the collection and select the sculptures to explore, we designed a simple selection widget based on a hierarchy of thumbnail-based scroll interfaces. The first level of hierarchy is a scroll bar containing images of the four statue categories: boxers, archers, warriors, and building models (see figure 3 left). The second level of hierarchy contains instead, for each group, the thumbnails associated to each sculpture model (see figure 3 right). Navigating and exploring the entire collection is immediate, and users can decide in any moment to explore a different sculpture model with a limited number of scroll and select operations.

Camera control. There exist a number of variations of the 2D multi-touch RST technique adapted for 3D interaction, enabling simultaneous control of Rotation, Scale and Translation with just two fingers. No real standard has been defined for $3 \mathrm{D}$ touch-based interaction, although the pervasiveness of touch-based devices, like smartphones or tablets, has made the general user very familiar with touch interfaces. We chose to map common 2D RST gestures, such as pinch-to-zoom and drag-to-pan to 3D analogue behaviours: 1-finger drag performs a rotation, 2-finger drag is mapped to co-planar translation, and pinch performs incremental zoom in/out. Interactive exploration of complex 3D models requires continuously switching between rotation, panning and zooming, which can be difficult for novice users to handle. For this reason, we have implemented an automatic centering pivot (see ACeViT [19]) which performs a stochastic sampling of the visible surface every time the user pans or zooms in order to determine the pivot. In this way, the rotation pivot is always centered on the part of the object which is currently visible, thus providing a natural and understandable rotational behavior.

Image-based navigation. Image-assisted exploration has been implemented in many ways in the literature, both as hotspots to highlight interesting positions, or as 3D viewpoints linked to the scene providing real images integrated into the $3 \mathrm{D}$ representation. Although these approaches are typically invasive, cluttering the display and distracting the user from the main task. Our approach is navigation-oriented, providing the user with a collection of context-based selection of best views 

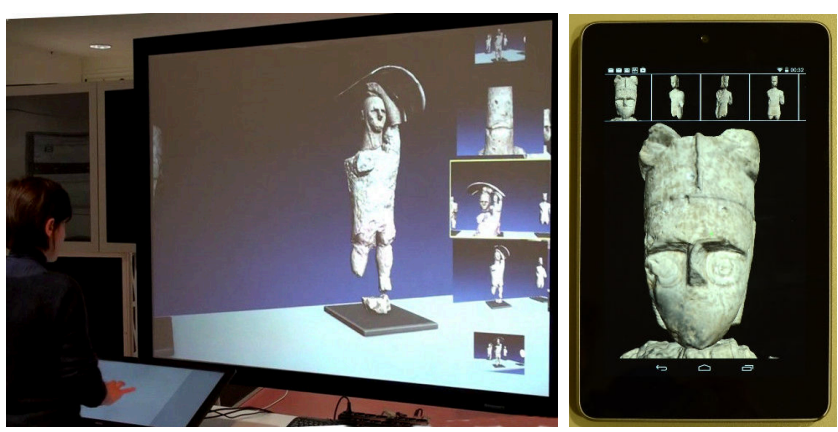

Fig. 4. Image-based selection. The user is presented with a set of interesting viewpoints near the current position. The same scroll view selector is employed for both the large display setup (see left), and the small screen mobile setup (see right).

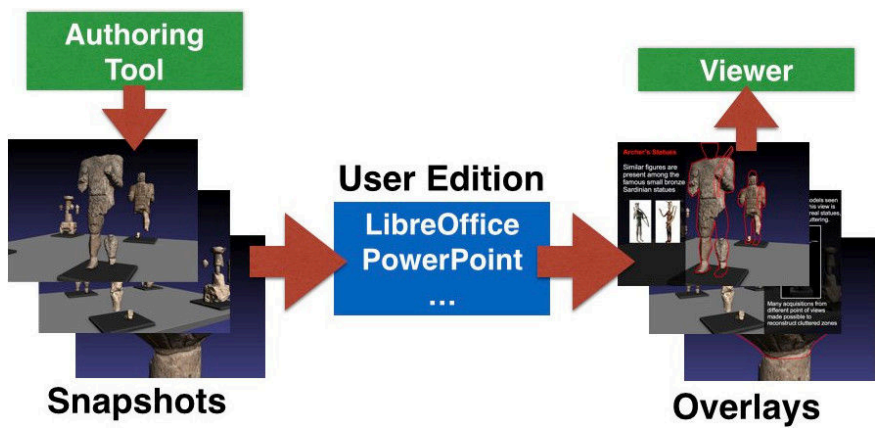

Fig. 5. Authoring overlays. Experts can create information overlays by selecting 3D points of interests, and by editing above associated views with popular image or presentation authoring software.

that can be used to travel to near interesting view points. This selection is based on an image-space distance function which selects a small number of views which are most similar in image-space to the current one. Figure 4 shows examples on how point-of-interest view selectors are integrated in a large display setup and on a small screen setup.

\section{INFORMATION PRESENTATION}

A variety of systems and standards for annotating and linking metadata to artworks exist [35], [36]. However, most of efforts are targeted to manage, link and spread information related to $2 \mathrm{D}$ artworks and images, by exploiting the capabilities of web and cloud computing [37], and adding spatial annotations to 3D models is still not considered an easy task for curators and cultural heritage experts. Our system was designed aiming at making it easy for archaeology experts and curators to quickly edit various kinds of informative content spatially associated to the sculptures. A follow-up work, not described in this paper, concerns techniques useful to provide more structure (e.g., temporal ordering) among annotations, as well as implicit presentation methods based on recommendation systems [31].

Information authoring. The authoring system is a version of the exploring application in which experts can navigate the sculpture collection and choose interesting views which they aim to comment, or where they want to draw some highlight or sketch, or add other kind of information (images, links). All selected views are saved in a database in form of pictures and the associated view transforms. Once the selection process is completed, the editing process consists of adding the annotations by employing the preferred image editing or presentation software to decorate the selected pictures. Each decorated images is then added to the database as overlay picture with transparent background (obtained with the removal of the original picture), and the information is ready to be displayed when users reach the associated view position. The authoring system is easy to use, fast and can be customized to add heterogeneous data as fields of database. Figure 5 shows a schematic diagram of the process of creating annotations, from selection of interesting view, to the information editing, and the resulting overlay picture to be displayed during the $3 \mathrm{D}$ navigation.

Overlay display. During the navigation, users can scroll a thumbnail-based point-of-interest selector showing the interesting viewpoints close to the area of the sculpture which is explored in that moment. The information overlays are displayed any time the user selects an interesting picture. Specifically, the system automatically leads the user to the selected view position and the information overlay appears to decorate that view, and it persists for a fixed amount of time (10-20 seconds according to the displayed information) or until the user decides to explore different parts of the sculpture or the collection by performing further interactions. In this way, users can easily get different kind of interesting information associated to the collection of artworks (see section VIII).

\section{SYSTEM INTEGRATION}

All the components of the architecture were designed to be easily adapted to work with different kind of displays and devices, ranging from smartphones to large high-resolution screens. We tested them in two different setups: one high end employing a large projector, and one for mobile devices.

High end setup. For this setup, we have preferred to decouple interaction and rendering, in order to permit the use of large displays for rendering. The touch device in charge of the user interface is located at a distance from the display enough to grant the user controlling the inspection with a whole view of the display, see Fig. 1. At the same time, this setup enables multiple users to watch the display without occlusion problems, which wouldn't be possible with an interactive wall display or a large touch monitor. The user interacts with a non co-located touch-based interface that provides very little visual information in order to not distract the user from the inspection task. The 3D navigation interface doesn't require co-located interaction, thus the user can abstract interaction just by dragging and pinching without any need to look at the touch monitor. At any time the user can scroll the imagebased view selector interface on the side, which shows the closest suggested point-of-interests (see Fig. 4).

Mobile setup. The majority of the requirements derived in the section IV are common both for the high end setup, and the mobile application, with the main difference being with respect to the display size and user interaction, besides performance issues. In this case, the co-located 3D navigation interface maps typical touch-based gestures, like dragging and pinching, into a behavior that appears natural and coherent with other standard mobile applications. Since there is no need for 
precise touch input it is easy for the user to concentrate on the inspection task, getting used to the interface just by trying typical gestures. The computation cost is only a small fraction of the frame rendering time, enabling the use of this solution in low performance devices. Due to the small size of the screen we have opted for a scroll widget that occupies a small part of the screen, allowing the user to select the target view with a simple click at any time, see Fig. 4. Additionally, since the list of proposed views is updated only when user stops interacting (i.e. not touching the screen), there are no distracting elements while the user is exploring the object. The rendering and user interface frameworks are shared between the various systems, providing a unified platform for distribution and rendering of large 3D models, see Fig. 2.

\section{IMPLEMENTATION AND RESULTS}

We have implemented the proposed approach both as a high-end system for museum installations, and as a mobile application, targeting mid-level hardware. Both systems share the data access components, using a NoSQL database (leveldb) for local data access and HTTP+Apache2 for networked access. The preprocessing step is also shared and enables processing with a performance of about $24 \mathrm{~K}$ triangles/second on an Intel Core i7 960 with $24 \mathrm{~GB}$ of RAM.

High-end system. The presented approach has been integrated into a reference system using OpenGL and GLSL for rendering, and Qt 4.7 for the interface. The system runs on a PC with Ubuntu Linux 12.10, with a Intel Core i7-3820 @ 3.6Ghz, 8GB of RAM and a NVIDIA GTX 680 GPU, sending the visual output to a $156 \mathrm{~cm} \times 250 \mathrm{~cm}$ back-projection screen through a 7500 ANSI Lumen DigitalProjection EVision WUXGA $(1920 \times 1200)$ projector. The user interface is controlled with a 27" DELL P2714T multi-touch screen, where very brief information is shown about the commands for controlling the navigation, only when there is no interaction, in order to attract the attention of the visitors. The multitouch screen was designed to be placed $180 \mathrm{~cm}$ away from the projection screen, allowing the user to see the whole screen in his field of view and, at the same time, to avoid obstacling other visitors to watch the display. The streaming performance under both high speed WIFI or wired networks provided refinement times below 5-10 seconds from cool start to a very close detail (i.e. an eye), requiring about 70MB download. During a typical exploration of the complex, users can gather lot of information and easily learn, for example, about the casual discovery of the sculptures (see Fig. 6(a)), the acquisition process (see Fig. 6(b)), their classification (see Fig. 6(c)), their peculiar and unique features (see Fig. 6(d)) as well as the similarities with respect to other artworks (see Fig. 6(g)) or to other existing building ruins (see Fig. 6(h)). Moreover, specific details can be highlighted on single sculptures, indicating measures (see Fig. 6(e)), specific carvings (see Fig. 6(f)), and the parts composing the models (see Fig. 6(i)). The museum setup has been permanently installed in two museums, one at the Museum Archeologico Nazionale di Cagliari, one at the Museum Civico di Cabras, where a large amount of visitors have been able to interactively explore the 3D representations of the various objects (i.e. statues and models) included in the collection in the same rooms containing the original statues. Furthermore, various custom versions of the museum systems have been installed in successful purely virtual temporary exhibitions visited by tens of thousands of people: Fiera Internazionale di Cagliari (May 2013), Museo Nazionale Preistorico Etnografico "Luigi Pigorini” in Rome (November 2014March 2015), and Museo Civico Archeologico in Milan (MayNovember 2015). From the reports obtained from all locations, the system has been tested extensively by people from all age ranges with successful results. The simplicity of the control metaphor, together with the high resolution rendering permit the visitors to inspect very close details of the objects that would be otherwise hard to observe on the real ones (i.e., small carvings). Additionally, the large display provides the correct sense of scale transmitting to the user the sensation of exploring an imposing artwork. Given the enormous success of the previous exhibitions, other custom versions of the museal setup are currently developed and ready to be installed for permanent exhibitions and for temporary exhibitions in highly relevant national and international Cultural Heritage events.

Mobile system. We have also implemented our approach as a Android 2.3+ application, using OpenGL ES 2.0 and GLSL for the rendering through a thin wrapper unifying both ES and desktop OpenGL APIs. Qt 5.2 has been used for handling UI events and GL context creation, also providing good portability amongst Android, Windows, Linux or iOS. The application was tested in a variety of devices covering mid-class and highclass hardware, a number of display sizes ranging from 4 " to 10 ", and a wide range of screen resolutions (i.e. $1024 \times 768$ to $1920 x 1080)$. The performance was always interactive with minor performance issues under maximum render load, that was palliated by adaptively lowering the maximum triangle budget when interacting in order to maintain interactive frame rates. On mobile devices, the WIFI performance is very heterogeneous varying heavily amongst devices, besides the different screen resolutions. Initial loading time is below 5 seconds, while additional 5-10s are required for a fully refined full view. In order to have a fully refined close view (i.e. an eye or some millimeter sized carvings) 40-60 seconds are required to download the required data (i.e. below $40 \mathrm{MB}$ for $1 \mathrm{Mpixel}$ resolution, below $70 \mathrm{MB}$ for $2 \mathrm{Mpixel}$ resolution). Under UMTS/HSPA connection, again the main factor is connection performance, so under a connection with a peak fetch performance of $3.4 \mathrm{Mbps}$ having a fully refined close view required 120-140 seconds. Nevertheless, thanks to the multiresolution approach within a few seconds a reasonable quality representation is available for inspection. A series of preliminary usability tests were performed in order to evaluate the system with positive results. Figure 7 shows the exploration of two high resolution sculptures of the complex on a OnePlus One smartphone, running Android OS, with a 5.5" Full HD screen, 3GB of RAM, and a Snapdragon 801 quad-core CPU, paired with an Adreno 330 GPU.

\section{CONCLUSIONS}

We have presented our visualization architecture for exploring extremely detailed annotated surface models, which has been developed for the valorization of an extraordinary collection of protostoric Mediterranean sculptures and it has been integrated both in mobile applications and in high end setups. The system successfully integrates network streaming, scalable multiresolution structures, and adaptive rendering techniques. The rendering framework has demonstrated its scalability by enabling interactive rendering of huge 3D models 


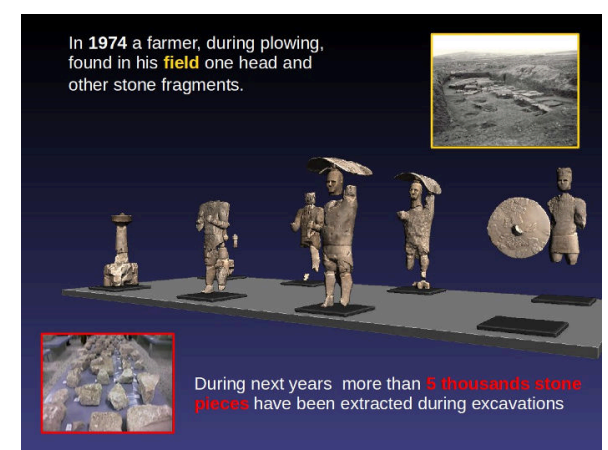

(a) Discovery of the sculpture complex

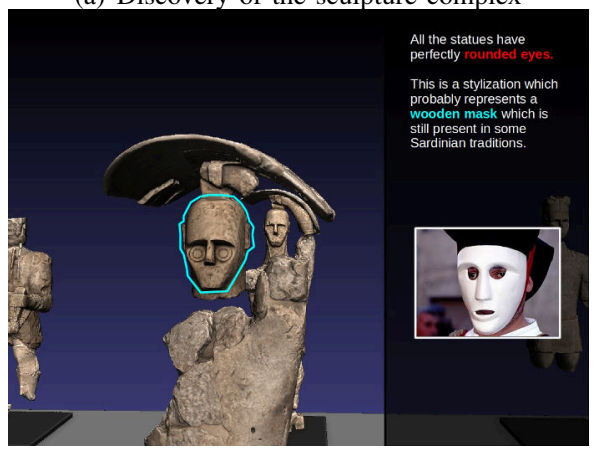

(d) Peculiar features

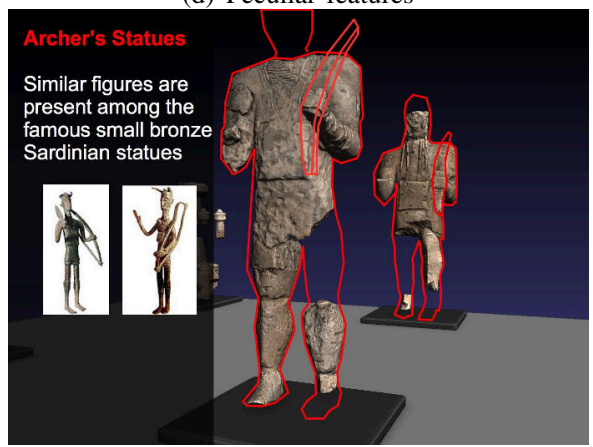

(g) Connections to other artworks

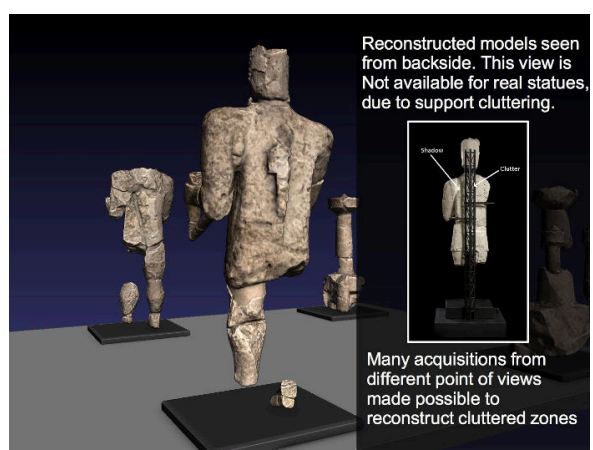

(b) Digital acquisition

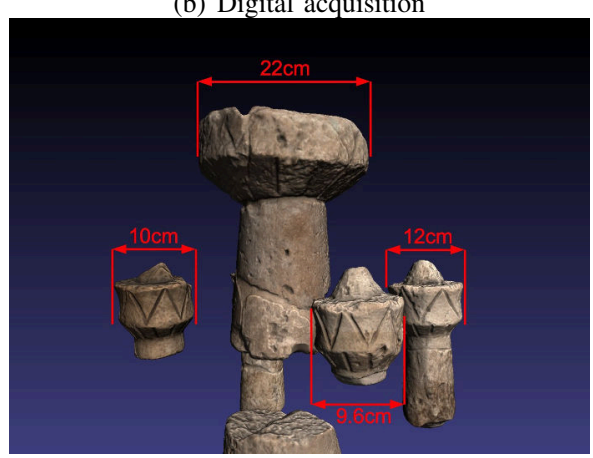

(e) Measure information

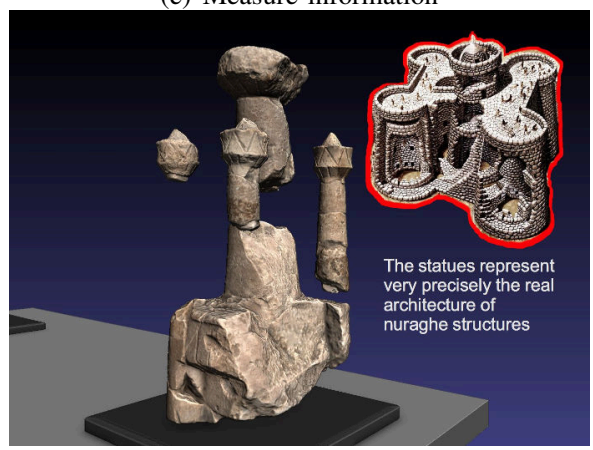

(h) Comparisons to other buildings

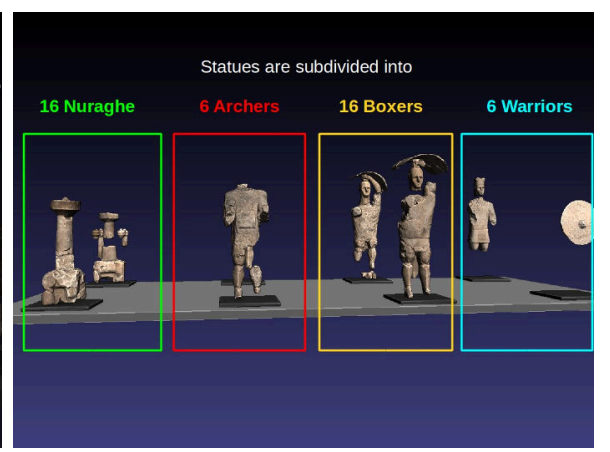

(c) Classification of sculptures

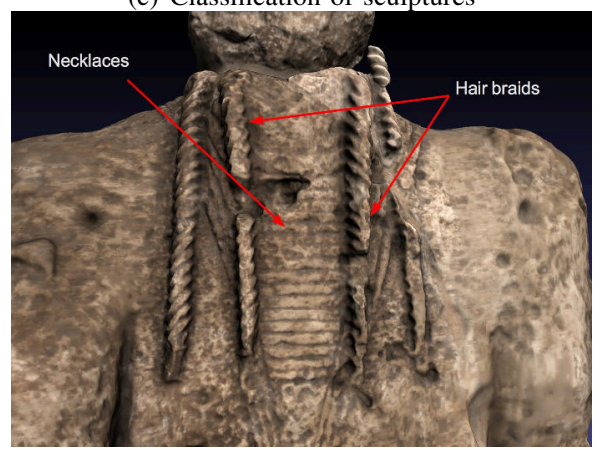

(f) Specific carvings

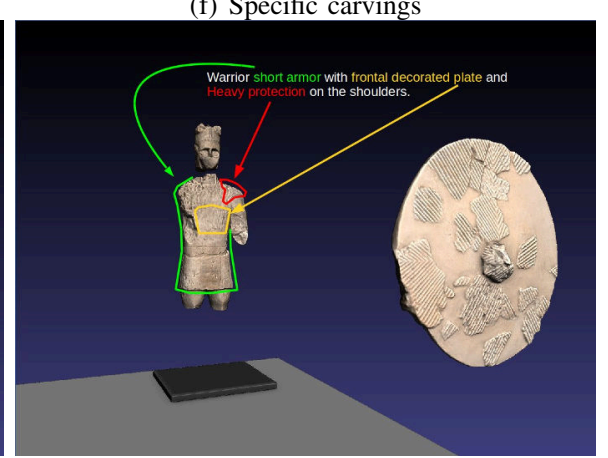

(i) Highlights of sculpture parts

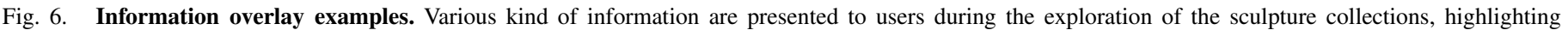
interesting artistic details or historical information.

both on high-end hardware and on embedded systems, with heavy restrictions both on rendering performance and resource availability. The 3D navigation user interface has been tested on a variety of display sizes permitting the user to naturally examine the artifacts, and smoothly transition from analyzing its shape to getting a very close view of a particular detail. The user interface has been implemented in large displays as a non co-located touch interface separated from the rendering display in order to provide both full field of view to the user, and low occlusion of the display to other visitors. On mobile devices, instead, the user interface is a co-located touch interface that maps to the well-known touch-based user interface typically present on this devices. Thanks to the automatic centering pivot the interaction with the touch screen can be abstracted allowing the user to focus on the rendered 3D model. Furthermore, a thumbnail-based selector widget allows users to easily discover interesting views of the sculpture collection, decorated with 2D overlays containing information previously authored by archaeology experts and curators. The large display setup has been tested in a variety of museum installations and exhibitions, while a cluster of user tests have been performed for the mobile version. Our current work concentrates on studying new ways to author and provide narrative content to users, as well as exploring the capabilities of new generation stereoscopic displays.

Acknowledgments. This work is partially supported by the EU FP7 Program under the DIVA project (290277), by RAS under projects BIGDATA and HELIOS, and by Soprintendenza per i Beni Archeologici per le Prov. di Cagliari ed Oristano (ArcheoCAOR).

\section{REFERENCES}

[1] M. Economou and E. Meintani, "Promising beginnings? evaluating museum mobile phone apps," in Proc. Rethinking Technology in Museums Conference, 2011, pp. 26-27.

[2] A. J. Karran, S. H. Fairclough, and K. Gilleade, "A framework for psychophysiological classification within a cultural heritage context using interest," ACM TOCHI, vol. 21, no. 6, p. 34, 2015.

[3] F. Bettio, E. Gobbetti, E. Merella, and R. Pintus, "Improving the digitization of shape and color of 3D artworks in a cluttered environment," in Proc. Digital Heritage, 2013, pp. 23-30. 

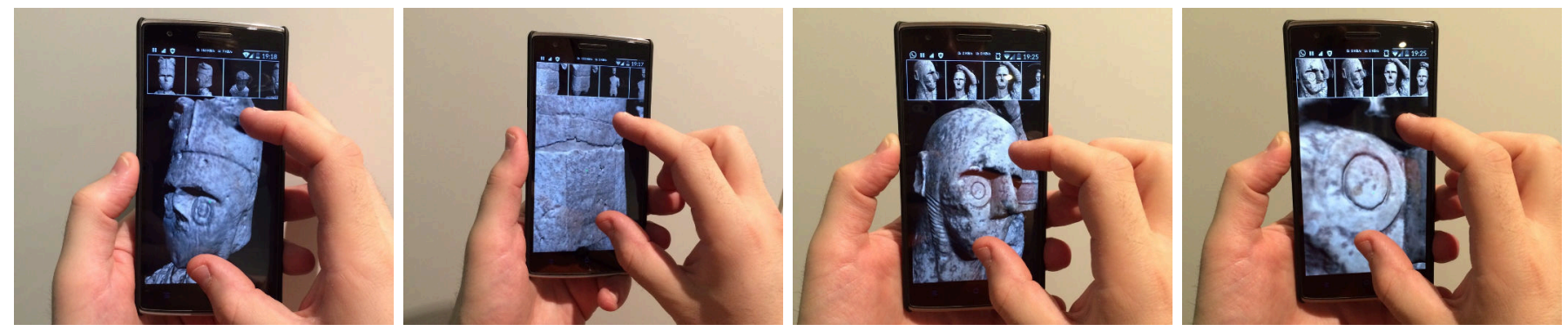

Fig. 7. Mobile device application. Exploration of highly detailed sculptures of the collection on a Android OnePlus One smartphone.

[4] F. Bettio, A. Jaspe Villanueva, E. Merella, F. Marton, E. Gobbetti, and R. Pintus, "Mont'e Scan: Effective shape and color digitization of cluttered 3D artworks," ACM JOCCH, vol. 8, no. 1, pp. 4:1-4:23, 2015.

[5] F. Marton, M. Balsa, F. Bettio, M. Agus, A. Jaspe Villanueva, and E. Gobbetti, "Isocam: Interactive visual exploration of massive cultural heritage models on large projection setups," JOCCH, 2014.

[6] S. Yoon, E. Gobbetti, D. Kasik, and D. Manocha, Real-time Massive Model Rendering, ser. Synthesis Lectures on Computer Graphics and Animation. Morgan and Claypool, August 2008, vol. 2.

[7] E. Gobbetti, D. Kasik, and S. Yoon, "Technical strategies for massive model visualization," in Proc. ACM SPM, 2008, pp. 405-415.

[8] A. Evans, M. Romeo, A. Bahrehmand, J. Agenjo, and J. Blat, "3D graphics on the web: A survey," Computers \& Graphics, vol. 41, pp. 43-61, 2014.

[9] E. Gobbetti, F. Marton, M. Balsa, F. Ganovelli, and M. Di Benedetto, "Adaptive Quad Patches: an adaptive regular structure for web distribution and adaptive rendering of 3D models," in Proc. ACM Web3D, 2012, pp. 9-16.

[10] M. Balsa, E. Gobbetti, F. Marton, R. Pintus, G. Pintore, and A. Tinti, "Interactive exploration of gigantic point clouds on mobile devices," in Proc. VAST, November 2012, pp. 57-64.

[11] M. Balsa, E. Gobbetti, F. Marton, and A. Tinti, "Compression-domain seamless multiresolution visualization of gigantic meshes on mobile devices," in Proc. ACM Web3D, June 2013, pp. 99-107.

[12] M. Balsa Rodríguez, E. Gobbetti, F. Marton, and A. Tinti, "Coarsegrained multiresolution structures for mobile exploration of gigantic surface models," in Proc. SIGGRAPH Asia Symposium on Mobile Graphics and Interactive Applications, 2013, pp. 4:1-4:6.

[13] F. Ramos, O. Ripolles, and M. Chover, "Efficient visualization of 3D models on hardware-limited portable devices," Multimedia Tools and Applications, vol. 73, no. 2, pp. 961-976, 2014.

[14] M. Di Benedetto, F. Ponchio, L. Malomo, M. Callieri, M. Dellepiane, P. Cignoni, and R. Scopigno, "Web and mobile visualization for cultural heritage," in $3 D$ Research Challenges in Cultural Heritage. Springer, 2014, pp. 18-35.

[15] P. Cignoni, F. Ganovelli, E. Gobbetti, F. Marton, F. Ponchio, and R. Scopigno, "Adaptive tetrapuzzles: efficient out-of-core construction and visualization of gigantic multiresolution polygonal models," $A C M$ $T O G$, vol. 23, no. 3, pp. 796-803, 2004.

[16] J. Jankowski and M. Hachet, "A survey of interaction techniques for interactive 3D environments," in Eurographics STAR, 2013.

[17] T. Boubekeur, "Shellcam: Interactive geometry-aware virtual camera control," in Proc. ICIP, 2014, pp. 4003-4007.

[18] D. R. Trindade and A. B. Raposo, "Improving 3D navigation in multiscale environments using cubemap-based techniques," in Proc. ACM SAC, 2011, pp. 1215-1221.

[19] M. Balsa Rodriguez, M. Agus, F. Marton, and E. Gobbetti, "HuMoRS: Huge models mobile rendering system," in Proc. ACM Web3D International Symposium, 2014, pp. 7-16.

[20] C. Andujar, A. Chica, and P. Brunet, "Cultural heritage: User-interface design for the Ripoll monastery exhibition at the National Art Museum of Catalonia," Computers and Graphics, vol. 36, no. 1, pp. 28-37, 2012.

[21] D.-S. Ryu, W.-K. Chung, and H.-G. Cho, "PHOTOLAND: a new image layout system using spatio-temporal information in digital photos," in Proc. ACM SAC, 2010, pp. 1884-1891.

[22] C. Jang, T. Yoon, and H.-G. Cho, "A smart clustering algorithm for photo set obtained from multiple digital cameras," in Proc. ACM SAC, 2009, pp. 1784-1791.

[23] J. a. Mota, M. J. Fonseca, D. Gonçalves, and J. A. Jorge, "Agrafo: a visual interface for grouping and browsing digital photos," in Proc. ACM AVI, 2008, pp. 494-495.

[24] P. Faraday and A. Sutcliffe, "Designing effective multimedia presentations," in Proc. ACM SIGCHI, 1997, pp. 272-278.

[25] H. Sonnet, S. Carpendale, and T. Strothotte, "Integration of 3D data and text: The effects of text positioning, connectivity, and visual hints on comprehension," in Proc. INTERACT, ser. LNCS, 2005, vol. 3585, pp. $615-628$

[26] J. Jankowski, K. Samp, I. Irzynska, M. Jozwowicz, and S. Decker, "Integrating text with video and 3D graphics: The effects of text drawing styles on text readability," in Proc. ACM SIGCHI, 2010, pp. 1321-1330.

[27] N. F. Polys, D. A. Bowman, and C. North, "The role of depth and gestalt cues in information-rich virtual environments," International Journal of Human-Computer Studies, vol. 69, no. 1-2, pp. 30-51, Jan. 2011.

[28] T. Götzelmann, P.-P. Vázquez, K. Hartmann, A. Nürnberger, and T. Strothotte, "Correlating text and images: Concept and evaluation," in Proc. Smart Graphics, Berlin, Heidelberg, 2007, pp. 97-109.

[29] J. Jankowski and S. Decker, "A dual-mode user interface for accessing 3D content on the world wide web," in Proc. WWW, 2012, pp. 10471056.

[30] M. Callieri, C. Leoni, M. Dellepiane, and R. Scopigno, "Artworks narrating a story: a modular framework for the integrated presentation of three-dimensional and textual contents," in Proc. ACM Web3D, June 2013, pp. 167-175.

[31] M. Balsa Rodriguez, M. Agus, F. Marton, and E. Gobbetti, "Adaptive recommendations for enhanced non-linear exploration of annotated 3D objects," Computer Graphics Forum, vol. 34, no. 3, pp. 41-50, 2015.

[32] C. Tronchetti and P. Van Dommelen, "Entangled objects and hybrid practices: colonial contacts and elite connections at monte prama, sardinia," Journal of Mediterranean Archaeology, vol. 18, no. 2, p. 183, 2005.

[33] F. Bettio, E. Gobbetti, F. Marton, A. Tinti, E. Merella, and R. Combet, "A point-based system for local and remote exploration of dense 3D scanned models," in Proc. VAST, October 2009, pp. 25-32.

[34] M. Hachet, J.-B. de la Rivière, J. Laviole, A. Coh, and S. Cursan, "Touch-based interfaces for interacting with $3 \mathrm{D}$ content in public exhibitions," IEEE CG\&A, vol. 33, no. 2, pp. 80-85, Mar. 2013.

[35] P. Szekely, C. A. Knoblock, F. Yang, X. Zhu, E. E. Fink, R. Allen, and G. Goodlander, "Connecting the smithsonian american art museum to the linked data cloud," in The Semantic Web: Semantics and Big Data, 2013, pp. 593-607.

[36] C. Dijkshoorn, L. Aroyo, G. Schreiber, J. Wielemaker, and L. Jongma, "Using linked data to diversify search results a case study in cultural heritage," in Knowledge Engineering and Knowledge Management, 2014, pp. 109-120.

[37] A. Isaac and B. Haslhofer, "Europeana linked open data-data. europeana. eu," Semantic Web, vol. 4, no. 3, pp. 291-297, 2013. 